\title{
Technology in Endodontics: How is it Improving Quality of Treatments?
}

\author{
Edit Xhajanka ${ }^{1}$, Massimo Giovarruscio ${ }^{2}$, Almira Isufi ${ }^{3}$, Luciano Pacifici ${ }^{4}$, Orlando Donfrancesco ${ }^{5}$, Andrea Pacifici ${ }^{6}$, \\ Shilpa Bhandi ${ }^{7}$, Federico Valenti Obino ${ }^{8}$
}

World Journal of Dentistry (2021): 10.5005/jp-journals-10015-1848

In the last decades, technology has improved dentistry and endodontics significantly, providing useful tools for better diagnosis and root canal treatment. ${ }^{1,2}$ In the first decade of the millennium, microscopy has been the game-changer in endodontics, while in the second decade, three-dimensional radiography (CBCT) has played this role. CBCT has proved to be clinically useful not only by improving diagnosis and treatment planning, especially in the most difficult cases, but also by the clinical visualization and understanding of anatomic complexities. ${ }^{3}$ In addition, CBCT is a fundamental device providing images for guided endodontics. Static guides and dynamic navigation proved to be effective in the treatment of calcified canals, and also helpful in more conservative access cavities both in non-surgical and surgical endodontics. ${ }^{4,5}$ Ultimately, CBCT has been thoroughly used to evaluate and compare different materials used in the therapies that involve dental pulp, such as dental pulp-capping agents and materials for regenerative endodontics, or the efficiency of novel procedures that could be introduced in the ordinary clinical practice. ${ }^{6-8}$

Moreover, in the last decade, other two new manufacturing technologies have been changed root canal instrumentation and obturation: the heat treatment of nickel-titanium rotary instruments, ${ }^{9,10}$ and the introduction of new obturation materials, including bioceramic endodontic sealers, providing new products aiming at improving performance, safety, and simplicity of endodontic treatments. ${ }^{11-13}$

Endodontics has been a "2-dimensional" specialty for nearly 100 years, due to the fact that traditional $2 \mathrm{D}$ radiographs only allowed a partial visualization of anatomy and canal trajectories. This was related to the buccal-lingual direction of the $X$-rays and the superimposition of different structures. ${ }^{14,15}$ Using CBCT, and ideally using dedicated software for 3D reconstruction, the real anatomy of each case can be visualized by the endodontist, including hidden curvatures, hidden confluences, calcifications, etc. ${ }^{3,16}$ This is a huge advantage not only in terms of proper diagnosis and treatment planning but also to reduce iatrogenic errors during instrumentation procedures. Hidden curvatures which always lead to increased instrumentation stress, if not properly recognized, may easily result in intracanal separation. ${ }^{3}$ According to this, the knowledge of the intracanal anatomy is crucial to ideally predict the combination of stresses acting on the instruments and to better select the more appropriate ones to reduce the probability of intracanal failure. ${ }^{17-19}$ For $>25$ years, endodontists have been fearing sudden, unexpected breakage of nickel-titanium rotary instruments. ${ }^{1,20,21}$ Nowadays, we can tell that the great majority of those failures were related to the poor clinical understanding of anatomy, and consequently unproper choice and use of the instruments in very stressful, usually hidden, complexities. ${ }^{22}$ Clinical
${ }^{1}$ Department of Faculty of Medical Dentistry, Medical University of Tirana, Albania

${ }^{2}$ Department of Endodontics, Faculty of Dentistry, Oral and Craniofacial Sciences, Guy's Dental Hospital, London, UK; Institute of Dentistry, IM Sechenov First Moscow State Medical University, Moscow, Russian Federation

$3,4,6,8$ Department of Oral and Maxillo Facial Sciences, 'Sapienza' University of Rome, Rome, Italy

${ }^{5}$ Department of Oral and Maxillo Facial Sciences, 'Sapienza' University of Rome, Rome, Italy; Department of Anatomy, Histology, Forensic Medicine and Orthopaedics, Sapienza University of Rome, Rome, Italy

${ }^{7}$ Department of Restorative Dental Sciences, College of Dentistry, Jazan University, Jazan, Kingdom of Saudi Arabia

Corresponding Author: Almira Isufi, Department of Oral and Maxillo Facial Sciences, 'Sapienza' University of Rome, Rome, Italy, Phone: +39 3287353196, e-mail: almiraisufi@yahoo.it

How to cite this article: Xhajanka E, Giovarruscio M, Isufi A, et al. Technology in Endodontics: How is it Improving Quality of Treatments? World J Dent 2021;12(5):355-356.

Source of support: Nil

Conflict of interest: None

understanding of anatomy in three dimensions, commonly defined as "3D endodontics", is, therefore, a breakthrough in the clinical approach to improving the safety and simplicity of instrumentation procedures. In surgical endodontics, a 3D approach does the same, allowing a less invasive procedure, and reducing risks of iatrogenic errors.

\section{References}

1. Gambarini G, Piasecki L, Miccoli G, et al. Classification and cyclic fatigue evaluation of new kinematics for endodontic instruments. Aust Endod J 2019;45(2):154-162. DOI: 10.1111/aej.12294.

2. Piasecki L, Carneiro E, da Silva Neto UX, et al. The use of microcomputed tomography to determine the accuracy of 2 electronic Apex locators and anatomic variations affecting their precision. J Endod 2016;42(8):1263-1267. DOI: 10.1016/j.joen.2016.04.024.

3. Gambarini G, Ropini P, Piasecki L, et al. A preliminary assessment of a new dedicated endodontic software for use with CBCT images to evaluate the canal complexity of mandibular molars. Int Endod J 2018;51(3):259-268. DOI: 10.1111/iej.12845.

4. Gambarini G, Galli M, Morese A, et al. Digital design of minimally invasive endodontic access cavity. App Sci (Switzerland) 2020;10(10):3513. DOI: 10.3390/app10103513.

5. Gambarini G, Galli M, Morese A, et al. Precision of dynamic navigation to perform endodontic ultraconservative access cavities:

() Jaypee Brothers Medical Publishers. 2021 Open Access This article is distributed under the terms of the Creative Commons Attribution 4.0 International License (https://creativecommons.org/licenses/by-nc/4.0/), which permits unrestricted use, distribution, and non-commercial reproduction in any medium, provided you give appropriate credit to the original author(s) and the source, provide a link to the Creative Commons license, and indicate if changes were made. The Creative Commons Public Domain Dedication waiver (http://creativecommons.org/publicdomain/zero/1.0/) applies to the data made available in this article, unless otherwise stated. 
a preliminary in vitro analysis. J Endod 2020;46(9):1286-1290. DOI: 10.1016/j.joen.2020.05.022.

6. ElSheshtawy AS, Nazzal H, El Shahawy Ol, et al. The effect of plateletrich plasma as a scaffold in regeneration/revitalization endodontics of immature permanent teeth assessed using 2-dimensional radiographs and cone beam computed tomography: a randomized controlled trial. Int Endod J 2020;53(7):905-921. DOI: 10.1111/iej. 13303.

7. Muruganandhan J, Sujatha G, Poorni S, et al. Comparison of four dental pulp-capping agents by cone-beam computed tomography and histological techniques-a split-mouth design ex vivo study. Appl Sci (Switzerland) 2021;11(7):3045.

8. Shetty $\mathrm{H}$, Shetty $\mathrm{S}$, Kakade A, et al. Three-dimensional qualitative and quantitative analyses of the effect of periradicular lesions on the outcome of regenerative endodontic procedures: a prospective clinical study. Clin Oral Investig 2021;25(2):691-700. DOI: 10.1007/ s00784-020-03583-z.

9. Gambarini G, Galli M, Di Nardo D, et al. Differences in cyclic fatigue lifespan between two different heat treated NiTi endodontic rotary instruments: WaveOne Gold vs EdgeOne Fire. J Clin Exp Dent 2019;11(7):e609-e613. DOI: 10.4317/jced.55839.

10. Grande NM, Plotino G, Silla E, et al. Environmental temperature drastically affects flexural fatigue resistance of nickel-titanium rotary files. J Endod 2017;43(7):1157-1160. DOI: 10.1016/j.joen.2017. 01.040 .

11. Gambarini G, Testarelli L, Pongione G, et al. Radiographic and rheological properties of a new endodontic sealer. Aust Endod J 2006;32(1):31-34. DOI: 10.1111/j.1747-4477.2006.00005.x.

12. Turk T, Cicconetti A, Di Nardo D, et al. Nonsurgical retreatment using regenerative endodontic protocols: a case report. J Contemp Dent Pract 2020;21(11):1275-1278. DOI: 10.5005/jp-journals-100242909.
13. Gambarini G, Testarelli L, Galli M, et al. The effect of a new finishing process on the torsional resistance of twisted nickel-titanium rotary instruments. Minerva Stomatol 2010;59(7-8):401-406.

14. Valenti-Obino F, Di Nardo D, Quero L, et al. Symmetry of root and root canal morphology of mandibular incisors: a cone-beam computed tomography study. J Clin Exp Dent 2019;11(6):e527-e533. DOI: 10.4317/jced.55629.

15. Quero L, Schianchi G, Obino FV, et al. Root canal morphology of lower lateral incisors: a CBCT in vivo study. G Ital Endod 2020;34(1):41-46.

16. Tonini $\mathrm{R}$, Xhajanka $\mathrm{E}$, Giovarruscio $\mathrm{M}$, et al. Print and try technique: 3D-printing of teeth with complex anatomy a novel endodontic approach. Appl Sci (Switzerland) 2021;11(4):1-15.

17. Gambarini G, Seracchiani M, Zanza A, et al. Influence of shaft length on torsional behavior of endodontic nickel-titanium instruments. Odontology 2020. DOI: 10.1007/s10266-020-00572-2.

18. Seracchiani M, Miccoli G, Di Nardo D, et al. Effect of flexural stress on torsional resistance of NiTi instruments. J Endodont 2020;47(3). DOI: 10.1016/j.joen.2020.10.011.

19. Seracchiani M, Miccoli $G$, Reda R, et al. A comprehensive in vitro comparison of mechanical properties of two rotary endodontic instruments. World J Dentis 2020;11(3):185-188. DOI: 10.5005/ jp-journals-10015-1729.

20. Miccoli G, Cicconetti A, Gambarini G, et al. A new device to test the bending resistance of mechanical endodontic instruments. Appl Sci (Switzerland) 2020;10(20):1-6. DOI: 10.3390/app10207215.

21. Gambarini G, Cicconetti A, Nardo DD, et al. Influence of different heat treatments on torsional and cyclic fatigue resistance of nickeltitanium rotary files: a comparative study. Appl Sci (Switzerland) 2020;10(16):5604.

22. Gambarini G, Seracchiani M, Piasecki L, et al. Measurement of torque generated during intracanal instrumentation in vivo. Int Endod 2019;52(5):737-745. DOI: 10.1111/iej.13042. 